\title{
Quantifier Elimination via Clause Redundancy
}

\author{
Eugene Goldberg and Panagiotis Manolios \\ Northeastern University, USA, \{eigold,pete\}@ccs.neu.edu
}

\begin{abstract}
We consider the problem of existential quantifier elimination for Boolean formulas in conjunctive normal form. Recently we presented a new method for solving this problem based on the machinery of Dependency sequents (D-sequents). The essence of this method is to add to the quantified formula implied clauses until all the clauses with quantified variables become redundant. A D-sequent is a record of the fact that a set of quantified variables is redundant in some subspace. In this paper, we introduce a quantifier elimination algorithm based on a new type of D-sequents called clause D-sequents that express redundancy of clauses rather than variables. Clause D-sequents significantly extend our ability to introduce and algorithmically exploit redundancy, as our experimental results show.
\end{abstract}

\section{INTRODUCTION}

In this paper, we consider elimination of quantifiers from formulas of the form $\exists X[F]$ where $F$ is a Boolean formula in conjunctive normal form (CNF). We will refer to such formulas as $\exists \mathrm{CNF}$. The Quantifier Elimination (QE) problem, is to find a quantifier-free $\mathrm{CNF}$ formula $G$ such that $G \equiv \exists X[F]$. The equivalence ' $\equiv$ ' is semantic. That is for every complete assignment $s$ to the non-quantified variables of $F$, the logical value of $G_{s}$ is equal to that of $\exists X\left[F_{s}\right]$. Here $F_{s}$ and $G_{s}$ are formulas $F$ and $G$ under assignment $s$.

The motivation for studying the QE problem is twofold. First, a QE algorithm can be used for solving many verification problems e.g. computing reachable states [5], [17]. Second, the methods developed for QE may come handy for other problems. For example, the machinery of Dependency sequents (D-sequents) [9], [10] that we continue developing in this paper can be used for SAT-solving [8].

In [9], [10], we developed a QE algorithm called $D D S$ (Derivation of D-Sequents) based on the following two ideas. The first idea is that adding resolvent clauses to formula $F$ eventually makes the clauses containing a variable of $X$ (we will call them $\boldsymbol{X}$-clauses) redundant. Let $H$ denote formula $F \wedge G$ where $G$ is the set of added resolvent clauses. For the sake of convenience, since a CNF formula can be considered as a set of clauses, we will use logical and set-based notation interchangeably. For example, formula $F \wedge G$ can also be written as $F \cup G$. The redundancy of $X$-clauses in $\exists X[H]$ means that $\exists X[H] \equiv \exists X\left[H \backslash H^{X}\right]$ where $H^{X}$ is the set of $X$-clauses of $H$. Since $H \backslash H^{X}$ does not depend on $X$, the quantifiers can be dropped. So the set of clauses $H \backslash H^{X}$ is a quantifier-free formula equivalent to $\exists X[H]$ and so to $\exists X[F]$. The second idea is to use a divide-and-conquer strategy. $D D S$ proves redundancy of $X$-clauses in subspaces and then merges the results of different branches.

A successful implementation of $D D S$ became possible only due to development of the machinery of D-sequents that was the main contribution of [9], [10]. A D-sequent is a record of the form $(\exists X[F], \boldsymbol{q}) \rightarrow Z$ where $\boldsymbol{q}$ is a partial assignment to variables of $F$ and $Z \subseteq X$. This D-sequent says that the variables of $Z$ are redundant in $\exists X[F]$ in subspace $\boldsymbol{q}$. The redundancy of variables of $Z$ means redundancy of all $X$ clauses containing a variable of $Z$. For the sake of brevity, in the following exposition we use the same symbol $F$ to denote the initial and the current $\mathrm{CNF}$ formula consisting of the initial clauses and resolvents. So symbol $F$ used in the D-sequent above is the current CNF formula.

$D D S$ keeps adding resolvent clauses to formula $F$ until Dsequent $(\exists X[F], \emptyset) \rightarrow X$ is derived stating that the variables of $X$ are redundant in formula $\exists X[F]$ globally. (This means that $F \backslash F^{X}$ is a solution to the QE problem.) The derivation of such D-sequent is achieved by generation of atomic Dsequents and using a resolution-like operation join. An atomic D-sequent is derived when redundancy of a variable of $X$ in a subspace can be trivially proved. Operation join is applied to D-sequents $\left(\exists X[F], \boldsymbol{q}^{\prime}\right) \rightarrow Z$ and $\left(\exists X[F], \boldsymbol{q}^{\prime \prime}\right) \rightarrow Z$ where $\boldsymbol{q}^{\prime}$ and $\boldsymbol{q}^{\prime \prime}$ contain opposite assignments to a variable $v$ of $F$. The result of join is a new D-sequent $(\exists X[F], \boldsymbol{q}) \rightarrow Z$ where $\boldsymbol{q}$ consists of all assignments of $\boldsymbol{q}^{\prime}, \boldsymbol{q}^{\prime \prime}$ but those to variable $v$.

The main contribution of this paper is the development of the machinery of a new type of D-sequents called clause D-sequents. A clause D-sequent is a record of the form $(\exists X[F], \boldsymbol{q}) \rightarrow R$ where $R \subseteq F^{X}$. It states that the $X$-clauses of $R$ are redundant in $\exists X[F]$ in subspace $\boldsymbol{q}$. Clause D-sequents can be used to express redundancy of any subset of $X$-clauses while D-sequents of [9] can do this only for some subsets of $X$-clauses. Namely, a D-sequent of [9] can express redundancy of a set $R \subseteq F^{X}$ only if $R$ is the set of all $X$-clauses containing variables from a set $Z$. (To distinguish new and old D-sequents we will refer to the latter as D-sequents based on variable redundancy.) For instance, a D-sequent based on variable redundancy cannot express the fact that a single $X$ clause $C$ is redundant in $\exists X[F]$ in subspace $\boldsymbol{q}$. Similarly to D-sequents based on variable redundancy, the machinery of clause D-sequents is based on a) derivation of atomic clause D-sequents, b) a resolution-like operation join and c) removing redundant $X$-clauses from the formula to guarantee the composability of D-sequents. The latter means that proving redundancy of sets of clauses $R^{\prime}$ and $R^{\prime \prime}$ independently implies that the set $R^{\prime} \cup R^{\prime \prime}$ is also redundant.

To show the advantages of clause D-sequents we describe a new QE algorithm called DCDS (Derivation of Clause DSequents). Development of $D C D S$ is another contribution of this paper. $D C D S$ can be viewed as an adaptation of $D D S$ to clause D-sequents. However, this adaptation is far from being trivial because clause D-sequents have new features that Dsequents based on variable redundancy do not. Using clause Dsequents is beneficial for at least two reasons. First, $D C D S$ has much more flexibility than $D D S$ in proving redundancy of $X$ clauses. Proving that a variable $v \in X$ is redundant in $\exists X[F]$ 
in subspace $\boldsymbol{q}$ by $D D S$ requires proving redundancy of all clauses of $F$ with variable $v$ at the same time. DCDS has no such restriction. Some clauses with variable $v$ may be proved redundant much later than the others. Second, the size of clause D-sequents is in general smaller than that of Dsequents based on variable redundancy. (The size of D-sequent $(\exists X[F], \boldsymbol{q}) \rightarrow R$ is the number of variables assigned in $\boldsymbol{q}$.) The reason is that proving redundancy of a clause is easier than that of a variable. This facilitates pruning the search space like derivation of shorter clauses does in SAT-solving.

This paper is structured as follows. In Section II, we give basic definitions. Simple cases of clause redundancy are discussed in Section III. Clause D-sequents are introduced in Section IV. Section V describes a new QE algorithm called $D C D S$. In Section VI, we explain $D C D S$ by a simple example. Experimental results are given in Section VII. Background is discussed in Section VIII, and conclusions are presented in Section IX.

\section{BASIC DEFINITIONS}

Definition 1: An $\exists \boldsymbol{C N F}$ formula is a quantified CNF formula of the form $\exists X[F]$ where $F$ is a CNF formula, and $X$ is a set of Boolean variables. If we do not explicitly specify whether we are referring to CNF or $\exists \mathrm{CNF}$ formulas, when we write "formula" we mean either a CNF or $\exists \mathrm{CNF}$ formula. Let $\boldsymbol{q}$ be an assignment, $F$ be a CNF formula, and $C$ be a clause. $\operatorname{Vars}(\boldsymbol{q})$ denotes the variables assigned in $\boldsymbol{q}$; $\operatorname{Vars}(F)$ denotes the set of variables of $F$; $\operatorname{Vars}(C)$ denotes the variables of $C$; and $\operatorname{Vars}(\exists X[F])=\operatorname{Vars}(F) \backslash X$.

We consider true and false as a special kind of clauses. A non-empty clause $C$ becomes true when it is satisfied by an assignment $\boldsymbol{q}$ i.e. when a literal of $C$ is set to true by $\boldsymbol{q}$. A clause $C$ becomes false when it is falsified by $q$ i.e. when all the literals of $C$ are set to false by $\boldsymbol{q}$.

Definition 2: Let $C$ be a clause, $H$ be a formula, and $\boldsymbol{q}$ be an assignment such that $\operatorname{Vars}(\boldsymbol{q}) \subseteq \operatorname{Vars}(H)$. Denote by $C_{\boldsymbol{q}}$ the clause equal to true if $C$ is satisfied by $\boldsymbol{q}$; otherwise $C_{\boldsymbol{q}}$ is the clause obtained from $C$ by removing all literals falsified by $\boldsymbol{q} . H_{\boldsymbol{q}}$ denotes the formula obtained from $H$ by replacing every clause $C$ of $H$ with $C_{\boldsymbol{q}}$. In the context of this paper, it is convenient to assume that clause $C_{q}$ equal to true remains in $H_{\boldsymbol{q}}$ rather than being removed from $H_{\boldsymbol{q}}$. We treat such a clause as redundant in $H_{\boldsymbol{q}}$ (see Proposition 1).

Definition 3: Let $G, H$ be formulas. We say that $G, H$ are equivalent, written $G \equiv H$, if for all assignments, $\boldsymbol{y}$, such that $\operatorname{Vars}(\boldsymbol{y}) \supseteq(\operatorname{Vars}(G) \cup \operatorname{Vars}(H))$, we have $G_{\boldsymbol{y}}=H_{\boldsymbol{y}}$ (notice that $G_{\boldsymbol{y}}$ and $H_{\boldsymbol{y}}$ have no free variables, so by $G_{\boldsymbol{y}}=H_{\boldsymbol{y}}$ we mean semantic equivalence). Observe that if $\operatorname{Vars}(\boldsymbol{q}) \subseteq$ $\operatorname{Vars}(\exists X[F])$, then $(\exists X[F])_{\boldsymbol{q}} \equiv \exists X\left[F_{\boldsymbol{q}}\right]$.

Definition 4: The Quantifier Elimination (QE) problem for $\exists \mathrm{CNF}$ formula $\exists X[F]$ consists of finding a CNF formula $G$ such that $G \equiv \exists X[F]$.

Definition 5: Let $Z$ be a set of variables. A clause $C$ of $F$ is called a $Z$-clause if $\operatorname{Vars}(C) \cap Z \neq \emptyset$. We denote by $F^{Z}$ the set of all $Z$-clauses of $F$.

Definition 6: Let $X$ be a set of Boolean variables, $F$ be a CNF formula and $R$ be a subset of $X$-clauses of $F$. The clauses of $R$ are redundant in CNF formula $F$ if $F \equiv(F \backslash R)$. The clauses of $R$ are redundant in $\exists \mathrm{CNF}$ formula $\exists X[F]$ if $\exists X[F] \equiv \exists X[F \backslash R]$. Note that $F \equiv(F \backslash R)$ implies $\exists X[F] \equiv \exists X[F \backslash R]$ but the opposite is not true.

\section{Simple CASES of Clause RedundanCy}

In this section, we describe three situations where clause redundancy can be trivially proved (Propositions 1, 2, 3).

Proposition 1: Let $\exists X[F]$ be an $\exists \mathrm{CNF}$ formula and $\boldsymbol{q}$ be an assignment to $\operatorname{Vars}(F)$ satisfying an $X$-clause $C$ of $F$. Then $C_{\boldsymbol{q}}$ is redundant in $\exists X\left[F_{\boldsymbol{q}}\right]$.

Due to lack of space we omit proofs. Note that proofs of non-trivial propositions of this paper are similar to those of [10] given for D-sequents based on variable redundancy.

Proposition 2: Let $\exists X[F]$ be an $\exists \mathrm{CNF}$ formula and $\boldsymbol{q}$ be an assignment to $\operatorname{Vars}(F)$. Let $C, C^{\prime}$ be two clauses of $F$. Let $C$ be falsified by $\boldsymbol{q}$ and $C^{\prime}$ be an $X$-clause. Then $C_{\boldsymbol{q}}^{\prime}$ is redundant in $\exists X\left[F_{\boldsymbol{q}}\right]$.

To formulate Proposition 3 below, we need to introduce a few definitions.

Definition 7: Let $C^{\prime}$ and $C^{\prime \prime}$ be clauses having opposite literals of exactly one variable $v \in \operatorname{Vars}\left(C^{\prime}\right) \cap \operatorname{Vars}\left(C^{\prime \prime}\right)$. The clause $C$ consisting of all literals of $C^{\prime}$ and $C^{\prime \prime}$ but those of $v$ is called the resolvent of $C^{\prime}, C^{\prime \prime}$ on $v$. Clause $C$ is said to be obtained by resolution on $v$. Clauses $C^{\prime}, C^{\prime \prime}$ are called resolvable on $v$.

Definition 8: A clause $C$ of a $\mathrm{CNF}$ formula $F$ is called blocked at variable $v$, if no clause of $F$ is resolvable with $C$ on $v$. The notion of blocked clauses was introduced in [15].

Proposition 3: Let $\exists X[F]$ be an $\exists \mathrm{CNF}$ formula and $\boldsymbol{q}$ be an assignment to $\operatorname{Vars}(F)$. Let $C$ be an $X$-clause of $F$ not satisfied by $\boldsymbol{q}$ and $v \in X$ be a variable of $C$ such that $v \notin$ $\operatorname{Vars}(\boldsymbol{q})$. Let clause $C_{\boldsymbol{q}}$ be blocked at $v$ in $F_{\boldsymbol{q}}$. Then $C_{\boldsymbol{q}}$ is redundant in $\exists X\left[F_{\boldsymbol{q}}\right]$.

\section{Dependency Sequents Based on Clause REDUNDANCY}

In this section, we define a new kind of dependency sequents (D-sequents) called clause D-sequents. In contrast to D-sequents of [9], clause D-sequents are based on the notion of clause redundancy. We describe operation join producing a new clause D-sequent from existing ones and introduce the notion of composable clause D-sequents.

\section{A. Definition of D-sequents}

Definition 9: Let $\exists X[F]$ be an $\exists \mathrm{CNF}$ formula. Let $\boldsymbol{q}$ be an assignment to $\operatorname{Vars}(F)$ and $R$ be a subset of $X$-clauses of $F$. A clause dependency sequent (clause D-sequent) has the form $(\exists X[F], \boldsymbol{q}) \rightarrow R$. It states that the clauses of $R_{\boldsymbol{q}}$ are redundant in $\exists X\left[F_{\boldsymbol{q}}\right]$.

From now on we will refer to clause D-sequents as just D-sequents unless we want to contrast clause D-sequents with those based on variable redundancy.

Example 1: Consider an $\exists \mathrm{CNF}$ formula $\exists X[F]$ where $F=C_{1} \wedge C_{2}, C_{1}=x \vee y_{1}$ and $C_{2}=\bar{x} \vee y_{2}$ and $X=\{x\}$. Let 
$\boldsymbol{q}=\left\{\left(y_{1}=1\right)\right\}$. Then clause $C_{1}$ is satisfied by $\boldsymbol{q}$ and according to Proposition 1, the D-sequent $(\exists x[F], \boldsymbol{q}) \rightarrow\left\{C_{1}\right\}$ holds. Since $F_{\boldsymbol{q}}=\left\{\right.$ true, $\left.C_{2}\right\}$, clause $C_{2}$ is blocked at variable $x$. So according to Proposition 3 , the D-sequent $(\exists x[F], \boldsymbol{q}) \rightarrow\left\{C_{2}\right\}$ holds.

According to Definition 9, a D-sequent holds with respect to a particular $\exists \mathrm{CNF}$ formula $\exists X[F]$. Proposition 4 below shows that this D-sequent also holds after adding to $F$ any set of resolvent clauses.

Proposition 4: Let $\exists X[F]$ be an $\exists \mathrm{CNF}$ formula. Let $\boldsymbol{q}$ be an assignment to $\operatorname{Vars}(F)$. Let $G$ be a CNF formula such that $F \Rightarrow G$. Then if $(\exists X[F], q) \rightarrow R$ holds, $(\exists X[F \wedge G], \boldsymbol{q}) \rightarrow R$ does too.

\section{B. Join Operation for D-sequents}

In this subsection, we introduce the operation of joining D-sequents (Definition 11).

Definition 10: Let $\boldsymbol{q}^{\prime}$ and $\boldsymbol{q}^{\prime \prime}$ be assignments in which exactly one variable $v \in \operatorname{Vars}\left(\boldsymbol{q}^{\prime}\right) \cap \operatorname{Vars}\left(\boldsymbol{q}^{\prime \prime}\right)$ is assigned different values. The assignment $\boldsymbol{q}$ consisting of all the assignments of $\boldsymbol{q}^{\prime}$ and $\boldsymbol{q}^{\prime \prime}$ but those to $v$ is called the resolvent of $\boldsymbol{q}^{\prime}, \boldsymbol{q}^{\prime \prime}$ on $v$. Assignments $\boldsymbol{q}^{\prime}, \boldsymbol{q}^{\prime \prime}$ are called resolvable on $v$.

Proposition 5: Let $\exists X[F]$ be an $\exists \mathrm{CNF}$ formula. Let Dsequents $\left(\exists X[F], \boldsymbol{q}^{\prime}\right) \rightarrow R$ and $\left(\exists X[F], \boldsymbol{q}^{\prime \prime}\right) \rightarrow R$ hold. Let $\boldsymbol{q}^{\prime}, \boldsymbol{q}^{\prime \prime}$ be resolvable on $v \in \operatorname{Vars}(F)$ and $\boldsymbol{q}$ be the resolvent of $\boldsymbol{q}^{\prime}$ and $\boldsymbol{q}^{\prime \prime}$. Then, D-sequent $(\exists X[F], \boldsymbol{q}) \rightarrow R$ holds too.

Definition 11: We will say that the D-sequent $(\exists X[F], \boldsymbol{q}) \rightarrow R$ of Proposition 5 is produced by joining D-sequents $\left(\exists X[F], \boldsymbol{q}^{\prime}\right) \rightarrow R$ and $\left(\exists X[F], \boldsymbol{q}^{\prime \prime}\right) \rightarrow R$ at $v$.

\section{Composable D-sequents}

In general, the fact that D-sequents $(\exists X[F], \boldsymbol{q}) \rightarrow R^{\prime}$ and $(\exists X[F], q) \rightarrow R^{\prime \prime}$ hold does not imply that $(\exists X[F], \boldsymbol{q}) \rightarrow R^{\prime} \cup R^{\prime \prime}$ holds. The reason is that redundancy of $R^{\prime}$ may be true only when clauses of $R^{\prime \prime}$ are in $F$ and vice versa. So, derivation of $(\exists X[F], \boldsymbol{q}) \rightarrow R^{\prime} \cup R^{\prime \prime}$ requires recursive reasoning. Proposition 6 below shows how to avoid recursive reasoning.

Let $\boldsymbol{q}$ and $s$ be assignments to a set of variables $Z$. Since $\boldsymbol{q}$ and $s$ are sets of value assignments to individual variables of $Z$, one can apply set operations to them. For instance, $s \subseteq \boldsymbol{q}$ means that $\boldsymbol{q}$ contains the value assignments of $\boldsymbol{s}$. Assignment $\boldsymbol{q} \cup \boldsymbol{s}$ consists of the value assignments that are in $\boldsymbol{q}$ or $\boldsymbol{s}$.

Proposition 6: Let $s$ and $\boldsymbol{q}$ be assignments to variables of $F$ where $s \subseteq \boldsymbol{q}$. Let D-sequents $(\exists X[F], \boldsymbol{s}) \rightarrow R^{\prime}$ and $\left(\exists X\left[F \backslash R^{\prime}\right], \boldsymbol{q}\right) \rightarrow R^{\prime \prime} \quad$ hold. Then D-sequent $(\exists X[F], \boldsymbol{q}) \rightarrow R^{\prime} \cup R^{\prime \prime}$ holds.

Definition 12: Let $\boldsymbol{q}^{\prime}$ and $\boldsymbol{q}^{\prime \prime}$ be assignments to a set of variables $Z$. We will say that $q^{\prime}$ and $q^{\prime \prime}$ are compatible if every variable of $\operatorname{Vars}\left(\boldsymbol{q}^{\prime}\right) \cap \operatorname{Vars}\left(\boldsymbol{q}^{\prime \prime}\right)$ is assigned the same value in $\boldsymbol{q}^{\prime}$ and $\boldsymbol{q}^{\prime \prime}$.

Definition 13: Let D-sequent $S^{\prime}$ be equal to $\left(\exists X[F], \boldsymbol{q}^{\prime}\right) \rightarrow R^{\prime}$ and $S^{\prime \prime}$ be equal to $\left(\exists X[F], \boldsymbol{q}^{\prime \prime}\right) \rightarrow R^{\prime \prime}$ where $\boldsymbol{q}^{\prime}$ and $\boldsymbol{q}^{\prime \prime}$ are compatible assignments to $\operatorname{Vars}(F)$. We will call $S^{\prime}$ and $S^{\prime \prime}$ composable if D-sequent $S$ equal to $\left(\exists X[F], \boldsymbol{q}^{\prime} \cup \boldsymbol{q}^{\prime \prime}\right) \rightarrow R^{\prime} \cup R^{\prime \prime}$
$/ / \boldsymbol{q}$ is an assignment to $\operatorname{Vars}(F)$

$/ / \Omega$ denotes a set of active D-sequents

// $\Phi$ denotes $\exists X[F]$

// If $D C D S$ returns clause nil (respectively a non-nil clause),

$/ / F_{\boldsymbol{q}}$ is satisfiable (respectively unsatisfiable)

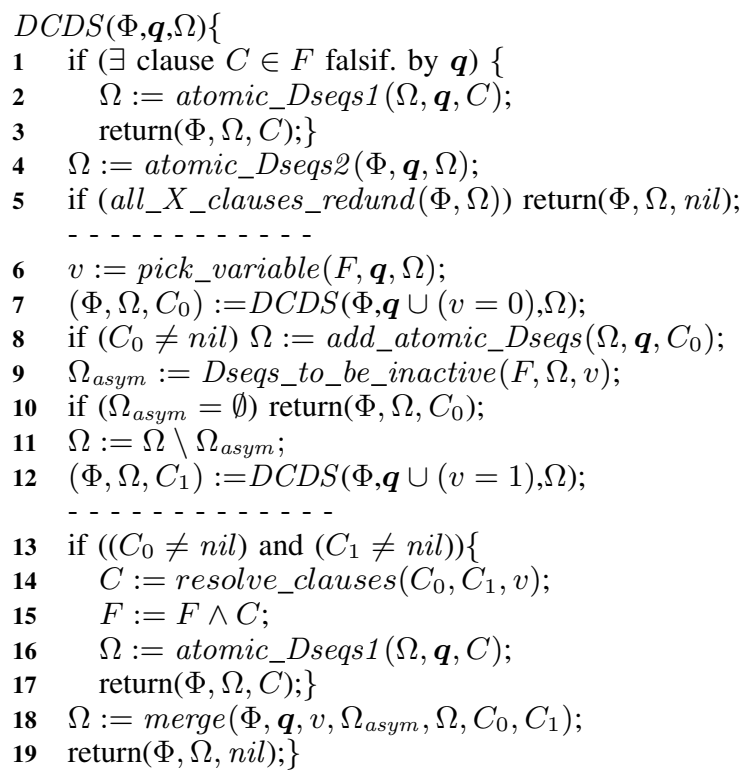

Fig. 1. $D C D S$ procedure

holds. From Proposition 6 it follows that $S^{\prime}, S^{\prime \prime}$ are composable if D-sequent $\left(\exists X\left[F \backslash R^{\prime}\right], \boldsymbol{q}^{\prime} \cup \boldsymbol{q}^{\prime \prime}\right) \rightarrow R^{\prime \prime}$ or $\left(\exists X\left[F \backslash R^{\prime \prime}\right], \boldsymbol{q}^{\prime} \cup \boldsymbol{q}^{\prime \prime}\right) \rightarrow R^{\prime}$ hold.

Although the QE algorithm of [9] derives composable Dsequents we did not explicitly use the notion of composability of D-sequents there. In this paper, we make this important notion more conspicuous.

\section{Algorithm DESCRIPTION}

In this section, we describe a QE algorithm called $\boldsymbol{D C D S}$ (Derivation of Clause D-Sequents). $D C D S$ derives D-sequents $(\exists X[F], \boldsymbol{q}) \rightarrow\{C\}$ stating the redundancy of $X$-clause $C_{\boldsymbol{q}}$ of $F_{q}$. From now on, we will use a short notation of Dsequents writing $s \rightarrow\{C\}$ instead of $(\exists X[F], s) \rightarrow\{C\}$. We will assume that the parameter $\exists X[F]$ missing in $s \rightarrow\{C\}$ is the current $\exists \mathrm{CNF}$ formula (with all resolvents added to $F$ ).

One can omit $\exists X[F]$ from D-sequents because from Proposition 4 it follows that $(\exists X[F], s) \rightarrow\{C\}$ holds no matter how many resolvent clauses are added to $F$. We will call Dsequent $s \rightarrow\{C\}$ active in subspace $\boldsymbol{q}$ if $\boldsymbol{s} \subseteq \boldsymbol{q}$. If $\boldsymbol{s} \rightarrow\{C\}$ is active in subspace $\boldsymbol{q}$, clause $C_{\boldsymbol{q}}$ is redundant in $\exists X\left[F_{\boldsymbol{q}}\right]$.

A description of $D C D S$ is given in Figure 1. $D C D S$ accepts an $\exists \mathrm{CNF}$ formula $\exists X[F]$ (denoted as $\Phi$ ), an assignment $\boldsymbol{q}$ to $\operatorname{Vars}(F)$ and a set $\Omega$ of D-sequents active in subspace $\boldsymbol{q}$ stating redundancy of some $X$-clauses in $\exists X\left[F_{\boldsymbol{q}}\right]$. To simplify description of $D C D S$, by $\boldsymbol{X}$-clauses of $\boldsymbol{F}_{\boldsymbol{q}}$ we also mean the $X$-clauses of $F$ satisfied by $\boldsymbol{q}$. On the contrary, an $X$-clause of $F$ falsified by $\boldsymbol{q}$ is not considered as an $X$-clause of $F_{\boldsymbol{q}} . D C D S$ returns a formula $\exists X[F]$ modified by resolvent clauses added to $F$ (if any), a set $\Omega$ of D-sequents active in subspace $\boldsymbol{q}$ that state redundancy of all $X$-clauses in $\exists X\left[F_{\boldsymbol{q}}\right]$ 
and a clause $C$. If $F_{\boldsymbol{q}}$ is unsatisfiable then $C$ is a clause of $F$ falsified by $\boldsymbol{q}$. Otherwise, $C$ is equal to nil meaning that no clause implied by $F$ is falsified by $\boldsymbol{q}$.

The active D-sequents derived by $D C D S$ are composable. That is if $s_{1} \rightarrow\left\{C_{1}\right\}, \ldots, s_{k} \rightarrow\left\{C_{k}\right\}$ are the active D-sequents of subspace $\boldsymbol{q}$, then the D-sequent $s^{*} \rightarrow\left\{C_{1}, \ldots, C_{k}\right\}$ holds where $s^{*}=s_{1} \cup \ldots \cup s_{k}$ and $\boldsymbol{s}^{*} \subseteq \boldsymbol{q}$. $D C D S$ achieves composability of D-sequents as follows. As soon as an $X$-clause $C_{\boldsymbol{q}}$ is proved redundant, it is marked and ignored by $D C D S$, which is equivalent to removing $C_{\boldsymbol{q}}$ from $F_{\boldsymbol{q}}$. So $D C D S$ guarantees that for every path of the search tree leading to a leaf, $X$-clauses are proved redundant in a particular order. (This order may be different for different paths.) This allows to avoid recursive reasoning where a clause $C_{\boldsymbol{q}}^{\prime}$ is used to prove redundancy of clause $C_{\boldsymbol{q}}^{\prime \prime}$ and vice versa. In turn, avoiding recursive reasoning guarantees composability of D-sequents.

A solution to the QE problem in subspace $\boldsymbol{q}$ is obtained by discarding all $X$-clauses from the CNF formula $F_{\boldsymbol{q}}$ of $\exists X\left[F_{\boldsymbol{q}}\right]$ returned by $D C D S$. To build a quantifier-free $\mathrm{CNF}$ formula equivalent to $\Phi$, one needs to call $D C D S$ with $\boldsymbol{q}=\emptyset, \Omega=\emptyset$.

\section{A. The Big Picture}

$D C D S$ consists of three parts separated in Figure 1 by dotted lines. In the first part (lines 1-5), DCDS builds atomic D-sequents i.e. D-sequents for $X$-clauses whose redundancy can be trivially proved. If all $X$-clauses are proved redundant in $\exists X\left[F_{\boldsymbol{q}}\right], D C D S$ terminates.

If some $X$-clauses are not proved redundant yet, $D C D S$ enters the second part of the code (lines 6-12). First, $D C D S$ picks a branching variable $v$ (line 6). Then it extends $\boldsymbol{q}$ by assignment to variable $v$ and recursively calls itself (line 7) starting the left branch of $v$. For the sake of clarity, we assume that $D C D S$ first explores assignment $v=0$. Once the left branch is finished, $D C D S$ extends $\boldsymbol{q}$ by $(v=1)$ and explores the right branch (line 12).

In the third part, $D C D S$ merges the left and right branches (lines 13-19). The result of this merging is proving every $X$ clause redundant in $\exists X\left[F_{\boldsymbol{q}}\right]$. For every $X$-clause $C_{\boldsymbol{q}}$ proved redundant in $\exists X\left[F_{\boldsymbol{q}}\right]$, the set $\Omega$ contains precisely one active D-sequent $s \rightarrow\{C\}$ where $s \subseteq \boldsymbol{q}$. As soon as $C_{\boldsymbol{q}}$ is proved redundant, it is marked and ignored until $D C D S$ enters a subspace $q^{\prime}$ where $s \nsubseteq q^{\prime}$ i.e. a subspace where Dsequent $s \rightarrow\{C\}$ becomes inactive. Clause $C_{\boldsymbol{q}^{\prime}}$ is unmarked in $F_{\boldsymbol{q}^{\prime}}$ signaling that $D C D S$ needs to derive a new D-sequent $\boldsymbol{s}^{\prime} \rightarrow\{C\}$ where $\boldsymbol{s}^{\prime} \subseteq \boldsymbol{q}^{\prime}$ stating the redundancy of $C_{\boldsymbol{q}^{\prime}}$.

\section{B. Building Atomic D-sequents}

Procedures atomic_Dseqs1 and atomic_Dseqs 2 are called by $D C D S$ to compute D-sequents for trivial cases of clause redundancy listed in Section III. We refer to such D-sequents as atomic. Procedure atomic_Dseqs 1 is called when formula $F_{\boldsymbol{q}}$ contains an empty clause $C_{\boldsymbol{q}}$ which means that clause $C$ of $F$ is falsified by $\boldsymbol{q}$. For every $X$-clause $C_{\boldsymbol{q}}^{\prime}$ of $F_{\boldsymbol{q}}$ that has no active D-sequent yet, atomic_Dseql generates a D-sequent $s \rightarrow\left\{C^{\prime}\right\}$. Here $s$ is the shortest assignment falsifying $C$.
atomic_Dseqs2 $(\Phi, \boldsymbol{q}, \Omega)\{$

$1 \Omega:=\Omega \cup \operatorname{Dseqs}($ new_satisf_clauses $(\Phi, \boldsymbol{q}, \Omega))$;

$2 \Omega:=\Omega \cup$ Dseqs (new_blocked_clauses $(\Phi, \boldsymbol{q}, \Omega)$ );

$3 \operatorname{return}(\Omega)$;

Fig. 2. atomic_Dseqs2 procedure

If $F_{\boldsymbol{q}}$ does not have an empty clause, procedure atomic_Dseqs 2 shown in Figure 2 is called. It builds Dsequents for $X$-clauses that became satisfied or blocked in $F_{q}$. Let $C$ be a clause satisfied by $q$. Then D-sequent $s \rightarrow\{C\}$ is generated where $s=(v=b), b \in\{0,1\}$ is the assignment to a variable $v$ satisfying $C$.

Let $C_{\boldsymbol{q}}$ be blocked in $F_{\boldsymbol{q}}$ at variable $v \in X$ that is not assigned yet. A D-sequent $s \rightarrow\{C\}$ stating redundancy of $C$ is built as follows. The fact that $C_{\boldsymbol{q}}$ is blocked at $v$ means that every clause $C^{\prime}$ of $F$ resolvable with $C$ on $v$ is either satisfied by $\boldsymbol{q}$ or $C_{\boldsymbol{q}}^{\prime}$ is proved redundant in $F_{\boldsymbol{q}}$. The assignment $s$ is a subset of assignments of $\boldsymbol{q}$ guaranteeing that $C^{\prime}$ remains satisfied by $s$ or $C_{s}^{\prime}$ remains redundant in $\exists X\left[F_{s}\right]$ and so $C_{s}$ is blocked at $v$ in $F_{s}$. If $C^{\prime}$ is satisfied by $\boldsymbol{q}$, then $s$ contains an assignment $(v=b), b \in\{0,1\}$ of $\boldsymbol{q}$ satisfying $C^{\prime}$. If $C^{\prime}$ is not satisfied but $C_{\boldsymbol{q}}^{\prime}$ is proved redundant in $\exists X\left[F_{\boldsymbol{q}}\right]$, then $s$ contains all assignments of $\boldsymbol{s}^{\prime}$ where $\boldsymbol{s}^{\prime} \subseteq \boldsymbol{q}$ and $\boldsymbol{s}^{\prime} \rightarrow\left\{C^{\prime}\right\}$ is the D-sequent of $\Omega$ stating redundancy of $C_{q}^{\prime}$.

\section{Selection of a Branching Variable}

Let $\boldsymbol{q}$ be the assignment $D C D S$ is called with. We will say that a variable $x$ of $X$ is redundant in $\exists X\left[F_{\boldsymbol{q}}\right]$ if $x$ is not assigned in $\boldsymbol{q}$ and every $\{x\}$-clause is proved redundant in $\exists X\left[F_{\boldsymbol{q}}\right]$. Denote by $X_{\text {red }}$ the variables proved redundant in $\exists X\left[F_{\boldsymbol{q}}\right]$. Let $Y=\operatorname{Vars}(F) \backslash X$. DCDS branches on free (i.e., unassigned) variables of $X$ and $Y$. Importantly, a free variable $x \in X \backslash \operatorname{Vars}(\boldsymbol{q})$ is picked for branching only if $x \notin X_{\text {red }}$ i.e. $D C D S$ does not branch on variables proved redundant.

Although Boolean Constraint Propagation (BCP) is not shown explicitly in Figure 1, it is included into the pick_variable procedure as follows: a) preference is given to branching on variables of unit clauses of $F_{\boldsymbol{q}}$ (if any); b) if $v$ is a variable of a unit clause $C_{\boldsymbol{q}}$ of $F_{\boldsymbol{q}}$ and $v$ is picked for branching, then the value falsifying $C_{\boldsymbol{q}}$ is assigned first to cause immediate termination of this branch.

To simplify merging results of the left and right branches, $D C D S$ first assigns values to variables of $Y$ (more details are given in Subsection V-E). This means that pick_variable never selects a variable $x \in X$ for branching, if there is a free variable of $Y$. In particular, BCP does not assign values to variables of $X$ if a variable of $Y$ is still unassigned.

\section{Switching from Left to Right Branch}

$D C D S$ prunes big chunks of the search space by not branching on redundant variables of $X$. One more powerful pruning technique of $D C D S$ discussed in this subsection is reducing the size of right branches.

Let $s \rightarrow\{C\}$ be a D-sequent of the set $\Omega$ computed by $D C D S$ in the left branch $v=0$ (line 7 of Figure 1). We will call this D-sequent symmetric in $v$, if $v$ is not assigned in $s$. Otherwise, this D-sequent is called asymmetric in $\boldsymbol{v}$. Notice that if $s$ is symmetric in $v$, the D-sequent $s \rightarrow\{C\}$ is active in the right branch and so $C_{\boldsymbol{q}_{1}}$ is redundant in $\exists X\left[F_{\boldsymbol{q}_{1}}\right]$ where 


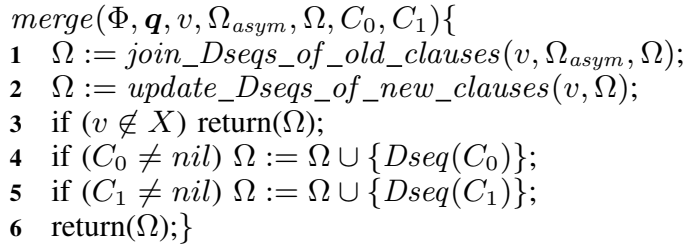

Fig. 3. merge procedure

$\boldsymbol{q}_{\mathbf{1}}=\boldsymbol{q} \cup\{(v=1)\}$. Denote by $\Omega_{\text {asym }}$ the subset of active D-sequents that are asymmetric in $v$. It is computed in line 9. Before exploring the right branch (line 12), the $X$-clauses of $F$ whose redundancy is stated by D-sequents of $\Omega_{\text {asym }}$ become non-redundant again. So the set of $X$-clauses to be considered in the right branch reduces to only those with $\mathrm{D}$-sequents from $\Omega_{\text {asym }}$. This allows to prune big parts of the search space. In particular, if $\Omega_{\text {asym }}$ is empty there is no need to explore the right branch. In this case, $D C D S$ just returns the results of the left branch (line 10). Pruning the right branch when $\Omega_{\text {asym }}$ is empty is similar to non-chronological backtracking well known in SAT-solving [16].

\section{E. Branch Merging}

Let $\boldsymbol{q}_{\mathbf{0}}=\boldsymbol{q} \cup\{(v=0)\}$ and $\boldsymbol{q}_{\mathbf{1}}=\boldsymbol{q} \cup\{(v=1)\}$. The goal of branch merging is to use solutions of the QE problem in subspaces $\boldsymbol{q}_{\mathbf{0}}$ and $\boldsymbol{q}_{\mathbf{1}}$ to produce a solution to the $\mathrm{QE}$ problem in subspace $\boldsymbol{q}$. If both $F_{\boldsymbol{q}_{0}}$ and $F_{\boldsymbol{q}_{1}}$ are unsatisfiable, this is done as described in lines 14-17 of Figure 1. In this case, the empty clauses $\left(C_{0}\right)_{q_{0}}$ and $\left(C_{1}\right)_{q_{1}}$ where $C_{0}, C_{1}$ are clauses returned in the left and right branches respectively are solutions to the QE in subspaces $\boldsymbol{q}_{\mathbf{0}}$ and $\boldsymbol{q}_{\mathbf{1}}$. The empty clause $C_{\boldsymbol{q}}$ where $C$ is the resolvent of $C_{0}$ and $C_{1}$ added to $F$ (line 15) is a solution to the QE problem in subspace $\boldsymbol{q}$. If, say, $v \notin \operatorname{Vars}\left(C_{1}\right)$ and so $C_{1}$ cannot be resolved on $v$, resolve_clauses (line 14) returns $C_{1}$ itself since $C_{1}$ is falsified by $\boldsymbol{q}$. In this case, no new clause is added to $F$. After $C$ is added, atomic_Dseqs 1 completes $\Omega$ by generation of atomic D-sequents built due to presence of a clause falsified by $\boldsymbol{q}$.

Suppose that at least one of formulas $F_{\boldsymbol{q}_{0}}$ and $F_{\boldsymbol{q}_{1}}$ is satisfiable. In this case, to finish solving the $\mathrm{QE}$ problem in subspace $\boldsymbol{q}$, one needs to make sure that every $X$-clause is proved redundant in $F_{\boldsymbol{q}}$. This means that every $X$-clause should have a D-sequent active in subspace $\boldsymbol{q}$ and hence symmetric in the branching variable $v$. This work is done by procedure merge shown in Figure 3 that consists of three steps. In the first step, merge takes care of D-sequents of "old" $X$ clauses that is the clauses that were present in $F$ at the time the value of $v$ was flipped from 0 to 1 . For every such $X$ clause, a D-sequent was derived in the left branch $v=0$. In the second step, merge processes new $X$-clauses that is $X$ clauses generated in the right branch $v=1$. No D-sequents were derived for such clauses in the branch $v=0$. In the third step, if, say, clause $C_{0}$ mentioned above is not equal to nil, a D-sequent is generated for $C_{0}$ if it is an $X$-clause.

In the first step, merge needs to update only D-sequents of $X$-clauses that became non-redundant in the right branch because their $D$-sequents got inactive there (such $D$-sequents form set $\Omega_{\text {asym }}$, see Subsection V-D). Let us denote this set of clauses as $G$. If a D-sequent of an $X$-clause $C$ from $G$ returned in the right branch is asymmetric in $v$, then join_Dseqs_of_old_clauses (line 1) replaces it with a D-sequent symmetric in $v$ as follows. Let $S_{0}$ and $S_{1}$ be the D-sequents derived in the left and right branches respectively that state the redundancy of $C_{\boldsymbol{q}_{0}}$ and $C_{\boldsymbol{q}_{1}}$. Then join_Dseqs_of_old_clauses joins $S_{0}$ and $S_{1}$ at $v$ producing a new D-sequent $S$. The latter states the redundancy of $C_{\boldsymbol{q}}$ and is symmetric in $v$. D-sequent $S_{1}$ is replaced in $\Omega$ with $S$.

Let $S_{1}$ be symmetric in $v$. If $F_{\boldsymbol{q}_{\mathrm{o}}}$ was unsatisfiable, then $S_{1}$ remains untouched. Otherwise, join_Dseqs_of_old_clauses does the following. Let $S_{1}$ be equal to $s \rightarrow\{C\}$. First, the right branch assignment $v=1$ is added to $s$, which makes $S_{1}$ asymmetric in $v$. Then $S_{1}$ is joined with $S_{0}$ at $v$ to produce a new D-sequent $S$ that is symmetric in $v$. $S$ replaces $S_{1}$ in $\Omega$. The reason one cannot simply keep $S_{1}$ in $\Omega$ untouched is as follows. As we mentioned above, the composability of Dsequents built by $D C D S$ is based on the assumption that for every path of the search tree, $X$-clauses are proved redundant in a particular order. It can be shown that using D-sequent $S_{1}$ in subspace $\boldsymbol{q}$ may violate this assumption and so break the composability of D-sequents.

Let $S$ be a D-sequent $s \rightarrow\{C\}$ derived in the right branch $v=1$ where $C$ was generated in this branch i.e. $C$ is a new clause. Such D-sequents are processed in the second step of merge by procedure update_dseqs_of_new_clauses (line 2). If $S$ is symmetric in $v$, it simply remains in $\Omega$ untouched. Otherwise, $S$ is updated by removing the assignment to $v$ from $s$. One can do this because the clause $C$ is implied by $F$ and has never been used in the left branch. So it can be considered as proved redundant in the left branch.

Finally, merge performs the third step (lines 3-5). Notice that if $v$ is not in $X$, then $C_{0}$ or $C_{1}$ is not an $X$-clause. This is because $D C D S$ assigns non-quantified variables before those of $X$ (see Subsection $\mathrm{V}$-C). So the last variable assigned in an $X$-clause is always a variable of $X$. Let us assume that $v \in X$ and $C_{0} \neq$ nil. (In the case $C_{1} \neq$ nil, merge works similarly.) Clause $\left(C_{0}\right)_{\boldsymbol{q}}$ is equal to the unit clause $v$. Notice that $F_{\boldsymbol{q}}$ does not contain a clause with literal $\bar{v}$ because this would mean that both $F_{\boldsymbol{q}_{0}}$ and $F_{\boldsymbol{q}_{1}}$ were unsatisfiable. So, $\left(C_{0}\right)_{\boldsymbol{q}}$ is blocked in $F_{\boldsymbol{q}}$ at variable $v$. Then an atomic D-sequent is built for $C_{0}$ as described in Subsection V-B.

\section{F. Correctness of DCDS}

Let $D C D S$ be called on formula $\Phi=\exists X[F]$ with $\boldsymbol{q}=\emptyset$ and $\Omega=\emptyset$. Here is an informal explanation of why $D C D S$ produces the correct result. First, new clauses of $F$ are produced by resolution and so are correct in the sense they are implied by $F$. In particular, if $F$ is unsatisfiable, $D C D S$ returns an empty clause that is a correct solution to the $\mathrm{QE}$ problem. Second, the atomic D-sequents built by $D C D S$ are correct. Third, new D-sequents produced by operation join are correct. Fourth, the D-sequents of individual clauses are composable. So when $D C D S$ returns to the root node of the search tree, it derives the correct D-sequent $(\exists X[F], \emptyset) \rightarrow F^{X}$. Hence, by removing $X$-clauses from $F$, one obtains a $\mathrm{CNF}$ formula that is a correct solution to the QE problem.

Proposition 7: DCDS is sound and complete.

\section{A RUN OF $D C D S$ ON A Simple Formula}

Let $\exists X[F]$ be an $\exists \mathrm{CNF}$ formula where $F=C_{1} \wedge C_{2}$, $C_{1}=\bar{y}_{1} \vee \bar{x}, C_{2}=y_{2} \vee x$ and $X=\{x\}$. To identify a particular 
$D C D S$ call we will use the corresponding assignment $\boldsymbol{q}$. For example, $D C D S_{\left(y_{1}=1, y_{2}=0\right)}$ means that the assignments $y_{1}=1$ and $y_{2}=0$ were made at recursion depths 0 and 1 respectively. Originally, assignment $\boldsymbol{q}$ is empty, so the initial call is $D C D S_{(\emptyset)}$. Figures 4, 5 show the work of $D C D S$. We use them to explain the algorithm of $D C D S$. For the sake of simplicity, in this example, we say that clause $C_{i}, i=1,2$ is blocked/redundant in $F_{\boldsymbol{q}}$ meaning that it is clause $\left(C_{i}\right)_{\boldsymbol{q}}$ that is blocked/redundant in $F_{\boldsymbol{q}}$.

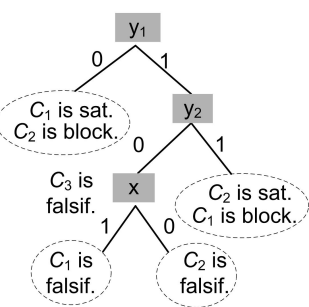

\section{Branching}

variables. Figure 4 shows the search tree built by $D C D S$. Recall that $D C D S$ branches on variables of $\operatorname{Vars}(F) \backslash X=\left\{y_{1}, y_{2}\right\}$ before those of $X$ (see Subsection V-C).

Leaves. The search tree of Figure 4 has four leaf nodes shown in dotted ovals. In each leaf node, either the $X$-clauses $C_{1}, C_{2}$ are

Fig. 4. Search tree built by $D C D S$ proved redundant or one of them is falsified. For example, $C_{1}$ is satisfied and $C_{2}$ is blocked, and hence $C_{1}, C_{2}$ are redundant, in leaf $\left(y_{1}=0\right)$ and clause $C_{1}$ is falsified in leaf $\left(y_{1}=1, y_{2}=0, x=1\right)$.

Generation of new clauses. $D C D S_{\left(y_{1}=1, y_{2}=0\right)}$ generates a new clause after branching on $x \cdot D C D S_{\left(y_{1}=1, y_{2}=0, x=1\right)}$ returns $C_{1}$ since it is falsified in $F_{\left(y_{1}=1, y_{2}=0, x=1\right)}$. Similarly, $D C D S_{\left(y_{1}=1, y_{2}=0, x=0\right)}$ returns $C_{2}$ since it is falsified in $F_{\left(y_{1}=1, y_{2}=0, x=0\right)}$. As described in Subsection V-E, in this case, $D C D S$ resolves clauses $C_{1}$ and $C_{2}$ on the branching variable $x$. The resolvent $C_{3}=\bar{y}_{1} \vee y_{2}$ is added to $F$.

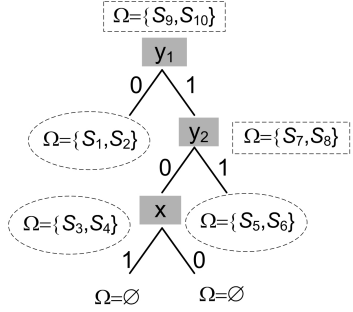

$S_{1}:\left(y_{1}=0\right) \rightarrow\left\{C_{1}\right\}, \quad S_{2}:\left(y_{1}=0\right) \rightarrow\left\{C_{2}\right\}$

$S_{3}:\left(\mathrm{y}_{1}=1, \mathrm{y}_{2}=0\right) \rightarrow\left\{C_{1}\right\}, S_{4}:\left(\mathrm{y}_{1}=1, \mathrm{y}_{2}=0\right) \rightarrow\left\{C_{2}\right\}$

$S_{5}:\left(y_{2}=1\right) \rightarrow\left\{C_{2}\right\}, \quad S_{6}:\left(y_{2}=1\right) \rightarrow\left\{C_{1}\right\}$

$S_{7}:\left(y_{1}=1\right) \rightarrow\left\{C_{1}\right\}, \quad S_{8}:\left(y_{1}=1\right) \rightarrow\left\{C_{2}\right\}$

$S_{9}: \varnothing \rightarrow\left\{C_{1}\right\}, \quad S_{10}: \varnothing \rightarrow\left\{C_{2}\right\}$

Fig. 5. Derivation of D-sequents
Generation of atomic Dsequents. Figure 5 describes derivation of D-sequents. The atomic D-sequents are shown in dotted ovals. (Dotted boxes show D-sequents obtained by operation join.) For instance, $C_{1}$ is satisfied by assignment $\left(y_{1}=0\right)$ and $C_{2}$ is blocked in $F_{\left(y_{1}=0\right)}$. So procedure atomic_Dseqs 2 called by $D C D S_{\left(y_{1}=0\right)}$ generates atomic D-sequents $S_{1}$ and $S_{2}$ equal to $\left(y_{1}=0\right) \rightarrow\left\{C_{1}\right\}$ and $\left(y_{1}=0\right) \rightarrow\left\{C_{2}\right\}$ respectively. The atomic D-sequents $S_{3}, S_{4}$ are derived by procedure atomic_Dseqsl called by $D C D S_{\left(y_{1}=1, y_{2}=0\right)}$. As we mentioned above, $D C D S_{\left(y_{1}=1, y_{2}=0\right)}$ adds clause $C_{3}=\bar{y}_{1} \vee y_{2}$ to $F$. This clause is empty in $F_{\left(y_{1}=1, y_{2}=0\right)}$ and so D-sequents $S_{3}, S_{4}$ equal to $s \rightarrow\left\{C_{1}\right\}, s \rightarrow\left\{C_{2}\right\}$ respectively are generated. Here $\boldsymbol{s}=\left(y_{1}=1, y_{2}=0\right)$ is the shortest assignment falsifying $C_{3}$.

Switching from left to right branch. Let us consider switching between branches by $D C D S_{(\emptyset)}$ where $y_{1}$ is picked for branching. The left branch of $\Omega_{(\emptyset)}$ returns D-sequents $S_{1}, S_{2}$ equal to $\left(y_{1}=0\right) \rightarrow\left\{C_{1}\right\}$ and $\left(y_{1}=0\right) \rightarrow\left\{C_{2}\right\}$ respectively.

Before starting the right branch $y_{1}=1, D C D S_{(\emptyset)}$ com- putes the set $\Omega_{(\emptyset)}^{a s y m}$ of D-sequents asymmetric in $y_{1}$. Since $S_{1}$ and $S_{2}$ contain an assignment to $y_{1}, \Omega_{(\emptyset)}^{\text {asym }}=\Omega_{(\emptyset)}$ and $D C D S_{(\emptyset)}$ removes $S_{1}, S_{2}$ from $\Omega$. So, before $D C D S_{\left(y_{1}=1\right)}$ is called, both $C_{1}$ and $C_{2}$ become non-redundant again.

Branch merging. Consider how branch merging is performed by $D C D S_{\left(y_{1}=1\right)}$. In the left branch $y_{2}=0$, D-sequents $S_{3}, S_{4}$ are derived that are asymmetric in $y_{2}$. In the right branch $y_{2}=1$, D-sequents $S_{5}, S_{6}$, also asymmetric in $y_{2}$, are produced. By joining $S_{3}$ and $S_{6}$ at $y_{2}$, D-sequent $S_{7}$ equal to $\left(y_{1}=1\right) \rightarrow\left\{C_{1}\right\}$ is derived. By joining $S_{4}$ and $S_{5}$ at $y_{2}$, D-sequent $S_{8}$ equal to $\left(y_{1}=1\right) \rightarrow\left\{C_{2}\right\}$ is derived. D-sequents $S_{7}, S_{8}$ state redundancy of $C_{1}, C_{2}$ in $\exists X\left[F_{\left(y_{1}=1\right)}\right]$.

Termination. When $D C D S_{(\emptyset)}$ terminates, $F=C_{1} \wedge C_{2} \wedge C_{3}$ where $C_{3}=\bar{y}_{1} \vee y_{2}$ and composable D-sequents $\emptyset \rightarrow\left\{C_{1}\right\}$ and $\emptyset \rightarrow\left\{C_{2}\right\}$ are derived. By dropping the $X$-clauses $C_{1}, C_{2}$ one obtains $C_{3} \equiv \exists X\left[C_{1} \wedge C_{2}\right]$.

Concluding remarks. Due to small size of $F$, some features of $D C D S$ are not exposed. For instance, the clause $C_{3}$ generated by $D C D S$ is not an $X$-clause. In general, $D C D S$ may produce new $X$-clauses whose redundancy one needs to prove along with the original $X$-clauses. Another consequence of using a small example is that D-sequents derived in every node has the form $s^{\prime} \rightarrow\left\{C_{1}\right\}$ and $s^{\prime \prime} \rightarrow\left\{C_{2}\right\}$ where $s^{\prime}=s^{\prime \prime}$. For larger formulas, assignments $s$ of active D-sequents $s \rightarrow\{C\}$ may be vastly different for different clauses $C$.

\section{EXPERIMENTAL RESULTS}

The objective of experiments was to compare the performance of $D D S$ and $D C D S$ on realistic examples and to give some comparison of D-sequent and BDD based algorithms. (A comparison of $D D S$ with SAT-based QE algorithms is given in [9].) Importantly, in the current implementations of $D D S$ and $D C D S$, D-sequents are not re-used. A D-sequent is discarded as soon as it is employed in a join operation.

We believe that reusing D-sequents will drastically boost the performance of both $D D S$ and $D C D S$ like conflict clause re-using speeds up SAT-algorithms. Re-using learned clauses in SAT-solving is beneficial because their involvement in BCP leads to new forced assignments. Re-using D-sequents gives the power of making "asymmetric" decision assignments. If, say, assignment $v=0$ makes a lot of learned D-sequents active, then this branch may be much easier to finish than branch $v=1$. Note that a forced assignment can be viewed as a special case of an asymmetric decision assignment where one of the two branches terminates immediately. Making asymmetric decision assignments leads to smaller search trees and so re-using D-sequents provides new exciting possibilities. However, tapping this power needs extra research. For that reason, we report experimental results for the algorithms without D-sequent re-using.

TABLE I. Results on examples solved by MC-DDS or MC-DCDS. The time limit is 2,000 s

\begin{tabular}{|l|l|l|l|}
\hline model checker & $M C-B D D$ & $M C-D D S$ & $M C-D C D S$ \\
\hline \#solved & 193 & 247 & $\mathbf{2 5 8}$ \\
\hline \#timeouts & 66 & 12 & 1 \\
\hline time for solved by all three (s.) & 9,080 & 11,293 & $\mathbf{1 , 6 9 8}$ \\
\hline
\end{tabular}

We applied $D D S$ and $D C D S$ to backward model checking. Our implementation was straightforward: $D D S$ and 
$D C D S$ were used to compute backward images until an initial state or a fixed point were reached. We will refer to these model checkers as $M C-D D S$ and $M C$ - $D C D S$. In experiments, we also used the BDD-based model checker incorporated into the latest version of a tool called PdTrav [22] (courtesy of Gianpiero Cabodi). We ran PdTrav in the backward model checking mode with ternary simulation turned off (as a non-BDD optimization). The other non-BDD optimizations, e.g. computation of the cone of influence, remained active because there was no way to switch them off. Since $D D S$ and $D C D S$ maintain a single search tree, we also forced PdTrav to represent the transition relation by a monolithic BDD. (A D-sequent based $\mathrm{QE}$ algorithm does not have to build a single search tree e.g. it can employ restarts. However, using restarts requires storing and re-using D-sequents.) We will refer to PdTrav with the options above as $M C-B D D$.

In the experiments, we ran $M C-D D S, M C-D C D S$ and $M C$ $B D D$ on 758 benchmarks of the HWMCC-10 competition [23] with the time limit of 2,000 seconds. $M C-B D D$ solved 374 benchmarks while $M C$-DDS and $M C-D C D S$ solved 247 and 258 benchmarks respectively. This is not surprising taking into account the maturity of current BDD algorithms and their re-using of learned information via subgraph hashing. An important fact however is that $M C-D C D S$ and even $M C$ $D D S$ solved many problems that $M C-B D D$ could not. So, in a sense, $M C-D D S$ with $M C-D C D S$ and $M C-B D D$ favored different subsets of benchmarks.

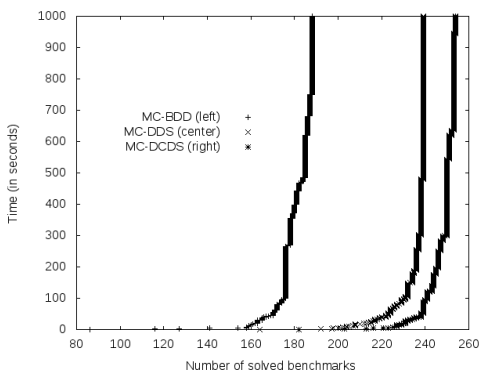

Fig. 6. Performance of model checkers on 259 examples solved by $M C-D D S$ or $M C$ $D C D S$

Table I shows the results of the three model checkers on the 259 benchmarks solved by $M C-D D S$ or $M C-D C D S \quad$ (i.e. favored by the two QE algorithms based on D-sequents). The second line of this table gives the number of benchmarks solved under 2,000s. The third line shows how many examples out of 259 were not solved in the time limit. The last line gives the total time in seconds for the benchmarks solved by all three model checkers. Table I shows that $M C-D C D S$ significantly outperformed $M C$-DDS. Besides, a large number of problems solved by $M C-D C D S$ were hard for $M C-B D D$. Figure 6 gives the performance of the three model checkers on the benchmarks solved by $M C-D D S$ or $M C-D C D S$ in terms of the number of problems finished in a given amount of time. $M C$ - $D C D S$ (the right line) consistently outperforms $M C$ - $D D S$ (the center line). Besides, on this set of benchmarks, both $M C-D D S$ and $M C-D C D S$ outperform $M C-B D D$ (the left line).

Results of all three model checkers on some concrete benchmarks from the 259 benchmark set are given in Table II. Symbol '*' marks benchmarks that were not solved in 2,000 s. The column iterations show the number of backward images computed by the algorithms before finding a bug or reaching a fixed point.
TABLE II. Some concrete examples. The time limit is 2,000s.

\begin{tabular}{|l|l|l|l|l|l|l|l|}
\hline benchmark & $\begin{array}{l}\text { \#lat- } \\
\text { ches }\end{array}$ & \#gates & $\begin{array}{l}\text { \#ite- } \\
\text { rati- } \\
\text { ons }\end{array}$ & bug & $\begin{array}{l}\text { MC- } \\
\text { BDD } \\
\text { (s.) }\end{array}$ & $\begin{array}{l}\text { MC- } \\
\text { DDS } \\
\text { (s.) }\end{array}$ & $\begin{array}{l}\text { MC- } \\
\text { DCDS } \\
\text { (s.) }\end{array}$ \\
\hline bj08amba4g5 & 36 & 13,637 & 4 & no & $*$ & 113 & 16 \\
\hline pdtvisbakery3 & 48 & 7,514 & 2 & yes & 1.3 & 12 & 5.1 \\
\hline texasifetch1p5 & 57 & 663 & 21 & yes & 1.5 & 368 & 96 \\
\hline visprodcellp01 & 78 & 2,885 & 5 & no & 19 & 7.9 & 2.3 \\
\hline texasparsesysp1 & 312 & 12,173 & 10 & yes & 0.7 & 231 & 41 \\
\hline bobmiterbm10r & 381 & 3,720 & 1 & yes & 0.7 & 0.1 & 0.1 \\
\hline pj2003 & 1175 & 15,384 & 3 & no & $*$ & $*$ & 252 \\
\hline bobsynthand & 3015 & 15,397 & 2 & no & 1.2 & 0.6 & 0.6 \\
\hline mentorbm1and & 4344 & 31,684 & 2 & no & $*$ & 1.8 & 1.4 \\
\hline
\end{tabular}

Table III sheds light on why $D C D S$ performs better than $D D S$. It shows results of applying both QE-algorithms to computing all bad states for some benchmarks (the first step of backward model checking). For either algorithm, we give the average size of atomic D-sequents and runtime. By the size of a D-sequent $s \rightarrow\{C\}$ we mean the number of variables assigned in $s$. To make a fair comparison we excluded the atomic D-sequents of length 1 generated when $X$ clauses got satisfied. Such D-sequents are not built by $D D S$.

TABLE III. Relation between average size of atomic D-sequents and runtime

\begin{tabular}{|l|l|l|l|l|}
\hline \multirow{2}{*}{ benchmark } & \multicolumn{2}{|l|}{$D D S$} & \multicolumn{2}{c|}{$D C D S$} \\
\cline { 2 - 5 } & $\begin{array}{l}\text { D-seq } \\
\text { size }\end{array}$ & $\begin{array}{l}\text { time } \\
\text { (s.) }\end{array}$ & $\begin{array}{l}\text { D-seq } \\
\text { size }\end{array}$ & $\begin{array}{l}\text { time } \\
\text { (s.) }\end{array}$ \\
\hline bc57sensorsp1 & 19 & 6.3 & 13 & 0.7 \\
\hline boblivea & 19 & 44 & 10 & 0.7 \\
\hline bobsmi2c & 7.4 & 96 & 2.0 & 9.4 \\
\hline cmugigamax & 20 & 3.6 & 9.4 & 3.2 \\
\hline csmacdp2 & 53 & 8.1 & 28 & 1.5 \\
\hline eijks344 & 6.0 & 7.5 & 2.5 & 1.6 \\
\hline pdtvissoap2 & 8.4 & 82 & 3.9 & 3.9 \\
\hline pj2006 & 7.3 & 47 & 1.1 & 0.4 \\
\hline
\end{tabular}

The results indicate that the size of atomic Dsequents derived by $D C D S$ was smaller. The reason is as follows. When an $X$-clause is blocked at a variable $v \in X$, $D C D S$ generates a D-sequent $s \rightarrow\{C\}$ where $s$ depends only on clauses that can be resolved with $C$ on a variable $v$. On the contrary, when a variable $v \in X$ is blocked, $D D S$ generates a D-sequent $s \rightarrow\{v\}$ where $s$ depends on all clauses that can be resolved on variable $v$. Such a D-sequent is, in general, much longer than $s \rightarrow\{C\}$.

\section{BACKGROUND}

The first practical QE algorithms were based on BDDs [3], [4]. Since we focus on SAT-based QE methods we do not discuss these algorithms here. The rest of QE algorithms can be roughly partitioned into two categories. The members of the first category employ various techniques to eliminate quantified variables of the formula one by one in some order [21], [1], [14], [7]. All these solvers face the same problem: there may not exist a good single order for variable elimination. This may lead to exponential growth of the size of intermediate formulas.

The solvers of the second category are based on enumeration of satisfying assignments [18], [12], [20], [13]. Since such assignments are, in general, "global" objects, it is hard for such solvers to follow the fine structure of the formula, e.g., such solvers are not compositional [9]. That is they cannot make use of the fact that formula $\exists X_{1}, X_{2}\left[F_{1} \wedge F_{2}\right]$ where $\operatorname{Vars}\left(F_{1}\right) \cap \operatorname{Vars}\left(F_{2}\right)=\emptyset$ and $X_{i} \subseteq \operatorname{Vars}\left(F_{i}\right), i=1,2$ is equivalent to $\exists X_{1}\left[F_{1}\right] \wedge \exists X_{2}\left[F_{2}\right]$.

In [9], we presented a $\mathrm{QE}$ algorithm called $D D S$ that employs the machinery of D-sequents based on redundancy 
of variables. In a sense, $D D S$ tries to take the best of both worlds. It branches and so can use different variable orders in different branches as the solvers of the second category. At the same time, in every branch, $D D S$ eliminates quantified variables individually as the solvers of the first category, which makes it easier to follow the formula structure. In particular, $D D S$ is compositional (as is $D C D S$ ).

Identification and removal of redundant clauses is used in preprocessing procedures of QBF-algorithms and SATsolvers [6], [2]. Redundant clauses are also identified in the inner loop of SAT-solving (inprocessing) [19]. These procedures identify unconditional clause redundancies by recognizing the situations where such redundancies can be easily proved.

Notice that any $X$-clause $C$ of a CNF formula $F$ can be made redundant in $\exists X[F]$ as follows. Let $v \in \operatorname{Vars}(C) \cap X$. Then $\exists X[F] \equiv \exists X[F \backslash\{C\} \cup G]$ where $G$ is the set of all clauses obtained by resolving $C$ with clauses of $F$ on $v$. In the context of SAT-solving, this fact has been established in [11], [19]. So to make $C$ redundant in $\exists X[F]$, one needs to add all the resolvents of $C$ with clauses of $F$ on a variable of $\operatorname{Vars}(C) \cap X$. Hence, one can potentially solve QE by gradually eliminating $X$-clauses (including the new $X$-clauses produced by resolution) in some order. Unfortunately, this approach has two fundamental problems. The first problem is similar to that of variable elimination. There may not exist a single good order for elimination of $X$-clauses. The second problem is that global elimination of $X$-clauses one by one may lead to looping even for small formulas. That is after elimination of a number of clauses one can return to a formula seen earlier e.g. to the original formula. This is because elimination of a clause is accompanied by adding new clauses and so removed clauses may reappear again.

$D C D S$ does not have the problems above. It is not limited by one global order because in different branches of the search tree redundancy of clauses is proved in different orders. $D C D S$ does not have the problem of looping because the branches of a search tree are ordered and the algorithm cannot visit the same state twice.

\section{CONCLUSIONS}

We continue to develop a calculus for solving propositional formulas with quantifiers based on the notion of dependency sequents (D-sequents). Previously, we introduced D-sequents recording redundancy of quantified variables. In this paper, we present a new type of D-sequents expressing redundancy of clauses containing quantified variables. The clause D-sequents are much more powerful that D-sequents based on variable redundancy. We use clause D-sequents to formulate a new algorithm of quantifier elimination called Derivation of Clause Dependency Sequents $(D C D S)$.

We experimentally compared $D C D S$ with a $\mathrm{QE}$ algorithm employing D-sequents based on variable redundancy in the context of model checking. The experiments showed that $D C D S$ significantly outperformed its counterpart. We also compared a model checker based on $D C D S$ with a BDDbased model checker. We found that there was a noticeable number of benchmarks where $D C D S$ outperformed its BDDbased counterpart. These results are very promising taking into account that the current version of $D C D S$ can be drastically improved e.g. by implementing D-sequent re-using.

\section{ACKNOWLEDGMENT}

This work was funded in part by NSF grant CCF-1117184. It was also supported in part by C-FAR, one of six centers of STARnet, a Semiconductor Research Corporation program sponsored by MARCO and DARPA.

\section{REFERENCES}

[1] P. Abdulla, P. Bjesse, and N. Een. Symbolic reachability analysis based on sat-solvers. TACAS-00, pages 411-425, 2000.

[2] A.Biere, F.Lonsing, and M.Seidl. Blocked clause elimination for qbf. CADE-11, pages 101-115, 2011.

[3] R. Bryant. Graph-based algorithms for Boolean function manipulation. IEEE Transactions on Computers, C-35(8):677-691, August 1986.

[4] P. Chauhan, E. M. Clarke, S. Jha, J.H. Kukula, H. Veith, and D. Wang. Using combinatorial optimization methods for quantification scheduling. CHARME-01, pages 293-309, 2001.

[5] E. Clarke, O. Grumberg, and D. Peled. Model checking. MIT Press, Cambridge, MA, USA, 1999.

[6] N. Eén and A. Biere. Effective preprocessing in sat through variable and clause elimination. In SAT, pages 61-75, 2005.

[7] E.Goldberg and P. Manolios. Sat-solving based on boundary point elimination. HVC-10, pages 93-111, 2010.

[8] E.Goldberg and P.Manolios. Checking satisfifiability by dependency sequents. Technical Report arXiv:1207.5014 [cs.LO], 2012.

[9] E.Goldberg and P.Manolios. Quantifier elimination by dependency sequents. In FMCAD-12, pages 34-44, 2012.

[10] E.Goldberg and P.Manolios. Quantifier elimination by dependency sequents. Technical Report arXiv:1201.5653 [cs.LO], 2012.

[11] A. V. Gelder. Propositional search with $k$-clause introduction can be polynomially simulated by resolution. In (Electronic) Proc. 5th Int'l Symposium on Artificial Intelligence and Mathematics, 1998.

[12] H.Jin and F.Somenzi. Prime clauses for fast enumeration of satisfying assignments to boolean circuits. DAC-05, pages 750-753, 2005.

[13] J.Brauer, A.King, and J.Kriener. Existential quantification as incremental sat. CAV-11, pages 191-207, 2011.

[14] J.R.Jiang. Quantifier elimination via functional composition. In Proceedings of the 21st International Conference on Computer Aided Verification, CAV-09, pages 383-397, 2009.

[15] O. Kullmann. New methods for 3-sat decision and worst-case analysis. Theor. Comput. Sci., 223(1-2):1-72, 1999.

[16] J. Marques-Silva and K. Sakallah. Grasp - a new search algorithm for satisfiability. In ICCAD-96, pages 220-227, 1996.

[17] K. McMillan. Symbolic Model Checking. Kluwer Academic Publishers, Norwell, MA, USA, 1993.

[18] K. McMillan. Applying sat methods in unbounded symbolic model checking. In Proc. of CAV-02, pages 250-264. Springer-Verlag, 2002.

[19] M.Järvisalo, M.Heule, and A.Biere. Inprocessing rules. IJCAR-12, pages 355-370, 2012.

[20] M.K.Ganai, A.Gupta, and P.Ashar. Efficient sat-based unbounded symbolic model checking using circuit cofactoring. ICCAD-04, pages 510-517, 2004.

[21] P. Williams, A. Biere, E. Clarke, and A. Gupta. Combining decision diagrams and sat procedures for efficient symbolic model checking. CAV-00, pages 124-138, 2000.

[22] http://fmgroup.polito.it/index.php/download/.

[23] HWMCC-2010 benchmarks, http://fmv.jku.at/hwmcc10/benchmarks.html. 